\title{
AN INELASTIC FINITE DISPLACEMENT FORMULATION OF THIN-WALLED MEMBERS
}

\author{
By Akio HASEGAWA*, Kithsiri K. LIYANAGE**, Masaru NODA*** \\ and Fumio NISHINO****
}

\begin{abstract}
The stiffness equation of linearized finite displacements for straight thin-walled members with inelastic material is derived. An arbitrary orthogonal coordinate system with a single reference point across the section need be introduced in the formulation, which is a clear distinction from the elasticity problem. Also distinct from the elastic analysis is a need to evaluate the magnitude of strains from time to time because of the dependence of the tangent modulus on strain levels. Illustrative examples are given to demonstrate the proposed method for the inelastic finite displacement analysis of spatial thin-walled members, with a simplified consideration on the effect of shear stresses.

Keywords: thin-walled members, finite displacement, inelasticity, tangent stiffness, spatial behaviour, constitutive equation.
\end{abstract}

\section{INTRODUCTION}

Generally, the stress and strain of the materials for structures undergoing large displacements do not follow a linear relation, even in the range of small strains. Hence, depending on the material, in many cases, it is necessary to formulate the problem reflecting inelasticity, in order to obtain realistic solutions. Going through the available literature, it can be seen that only a few studies have incorperated the effect of material inelasticity into the nonlinear finite displacement analysis of thin-walled structures.

Among those who treated the problem with inelastic material are Rajasekaran and Murray ${ }^{11.2)}$ who presented the beam equations referred to two arbitrary points fixed to the cross-section. A recent paper by Sakimoto et al. ${ }^{3)}$ gives an incremental formulation for the nonlinear behaviour of thin-walled inelastic members and frames with open cross-section, referring all the quantities to a single point on the cross-section. The aim of the present study is to develop a more general but rather simplified scheme for the nonlinear finite displacement analysis of thin-walled beams and frames with inelastic material.

Once some part of the thin-walled cross-section starts to develop inelasticity, the centroid and the shear center as well as the principal axes of the section change continuously with the increasing load. Hence there seems neither advantage in adopting such two special reference points, nor in making use of the properties of the principal axes. Rather than this, it is inevitable to introduce an arbitrary orthogonal coordinate system with a single reference point across the section, which makes a clear distinction from the elsticity

* Member of JSCE, Dr. Eng., Associate Professor, Division of Structural Engineering and Construction, Asian Institute of Technology (G. P. O. Box 2754, Bankok 10501, Thailand), on leave from University of Tokyo

** Dr. Eng., Lecturer, Department of Civil Engineering, University of Peradenia (Peradenia, Sri Lanka), formerly Graduate Student, Department of Civil Engineering, University of Tokyo

*** B. Eng., Graduate Student, Department of Civil Engineering, University of Tokyo (Bunkyo-ku, Tokyo)

**** Member of JSCE, Ph. D., Professor, Department of Civil Engineering, University of Tokyo (Bunkyo-ku, Tokyo) 
problem. Also distinct from the elastic analysis is a need to evaluate the magnitude of strains from time to time because of the dependence of the tangent modulus on strain levels. This paper is intended to give a straightforward and comprehensive formulation relating to those aspects. Noting that the axial stress is a major concern compared with the shear stress and the strain reversal does not play an influential role on the behaviour within the framework of the beam mechanics, however, the constitutive equation of material is assumed in a rather simpler fashion in the present study to avoid complication while keeping the essential aspects in mind.

\section{CONSTITUTIVE RELATIONS}

For the reason stated in INTRODUCTION, the axial stress is considered simply as a function of axial strain only, as given by

$$
\sigma_{x x}^{0}=f\left(e_{x x}^{0}\right)
$$

in which $\sigma_{x x}^{0}$ and $e_{x x}^{0}$ are the axial stress and strain respectively, and also for simplicity, the tangent shear modulus after yielding is assumed to be reduced by the same ratio as that for axial stress, and thus

$$
\frac{\partial \sigma_{s x}^{0}}{\partial e_{s x}^{0}}=2 \frac{G}{E} \frac{\partial_{x x}^{0}}{\partial e_{x x}^{0}}
$$

in which $E$ and $G$ are the modulus of elasticity and the shear modulus of the unstressed material with $\sigma_{s x}^{0}=$ $2 G e_{s x}^{0}$, respectively, and $\partial \sigma_{x x}^{0} / \partial e_{x x}^{0}$ for the respective location of the cross-section at the stressed stage can be determined by the relation given in Eq. $(1 \cdot \mathrm{a})$ as a function of the axial strain. It is noted that Eq. $(1 \cdot b)$ is newly introduced in this paper, but may not be exact nor consisitent in the sense of the rigorous treatise of the two-dimensional plasticity. Nevertheless, this assumption is worth to be introduced for a simpler formulation. When the actual inelastic behaviour of real structures is of primary interest, some modifications need to be considered within the frame work of the present formulation.

\section{GENERAL STIFFNESS OF THIN-WALLED INELASTIC MEMBERS}

A right hand cartesian coordinate system $(x, y, z)$ is being used in this study, as shown in Fig. 1 with $x$ along the member axis, and $y$ and $z$ being the original principal axes, with the origin at the centroid of the section at the unstressed state. In addition, for explanation purposes, another orthogonal set of coordinates $(x, n, s)$ is introduced, with $\mathrm{s}$ being the length coordinate measured along the middle surface of the thin-wall, starting from an arbitrary origin. Variables $u, v$, and $w$ are the incremental displacements in the directions $x, y$, and $z$ respectively, while $\phi$ is the incremental rotation of the cross-section. Variable $\omega$ is the normalized unit warping with respect to the centroid at the unstressed state.

Starting from the same assumptions and notations as introduced in Reference 4) except that for linear elasticity of the material, the incremental strain-displacement relations measured from the reference state

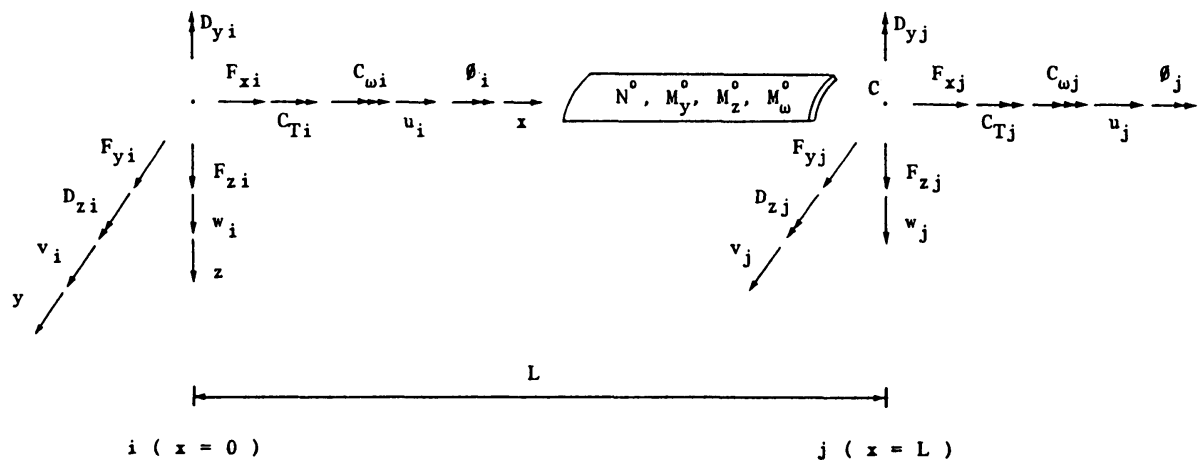

Fig. 1 Generalized Forces and Displacements. 
can be given in the form

$$
\begin{aligned}
& e_{x x}=u^{\prime}-v^{\prime \prime}(y-z \phi)-w^{\prime \prime}(z+y \phi)-\phi^{\prime \prime} \omega+\frac{1}{2}\left[\left(v^{\prime}\right)^{2}+\left(w^{\prime}\right)^{2}+\left(y^{2}+z^{2}\right)\left(\phi^{\prime}\right)^{2}\right] \\
& e_{s x}=\frac{1}{2} \theta \phi^{\prime}
\end{aligned}
$$

and

$e_{s s}=e_{n n}=e_{n s}=e_{x n}=0$

It should be noted that the right hand side of Eq. $(2 \cdot a)$ is not identical to that of Eq. ( 6$)$ in Reference 4), due to the reason that all the displacement quantities in Eq. $(2 \cdot a)$ are referred to a single point on the cross section, whereas, in Reference 4), some quantities are referred to the cross-sectional centroid and the others to the shear center. As far as nonlinear elastic materials are concerned, there seems no advantage in such a treatment, owing to the fact that the centroid as well as the shear center change continuously with the load. Because of this reason, in the present formulations, all displacement quantities are referred to a single point on the cross-section. As a matter of convenience, the cross-sectional centroid at the unstressed state is selected as the reference point. It should be noted that unit warping $\omega$ also is defined with respect to the centroid.

In the same way as in Reference 4), consider a uniform thin-walled beam element in equilibrium at an arbitrary reference state under a set of applied loads. The current displacement derivatives of the element at the reference state $\varepsilon^{0}\left[=\left(u^{0}\right)^{\prime}\right], \quad x_{y}^{0}\left[=-\left(v^{0}\right)^{\prime \prime}\right], \quad x_{z}^{0}\left[=-\left(w^{0}\right)^{\prime \prime}\right]$ and $x_{\omega}^{0}\left[=-\left(\phi^{0}\right)^{\prime \prime}\right]$ are supposed to be already known and assumed to be constant throughout the element.

The virtual work equation for the incremental step based on the linearized finite displacemnent formulation can be derived by the same procedure as in Reference 4) in the form

$$
\int_{v}\left(\sigma_{i j}^{0} \delta e_{i j}^{2}+\sigma_{i j}^{1} \delta e_{i j}^{1}\right) d v-\int_{V} T_{i} \delta u_{i} d s=0
$$

also using the same notations as in Reference 4).

Noting the relation as

$$
\sigma_{x x}=\frac{\partial \sigma_{x x}^{0}}{\partial e_{x x}^{0}} e_{x x} \text { at } e_{x x}^{0} \text { i. e. } \quad \Delta \sigma_{x x}^{0}=\frac{\partial \sigma_{x x}^{0}}{\partial e_{x x}^{0}} \Delta e_{x x}^{0}
$$

substitution of the strain-displacement relations (2) into Eq. (3) leads to

$$
\begin{aligned}
& \int_{v}\left[\sigma_{x x}^{0} \delta\left\{z v^{\prime \prime} \phi-y w^{\prime \prime} \phi+\frac{1}{2}\left[\left(v^{\prime}\right)^{2}+\left(w^{\prime}\right)^{2}+\left(\phi^{\prime}\right)^{2}\left(y^{2}+z^{2}\right)\right]\right\}\right. \\
& +\left(\partial \sigma_{x x}^{0} / \partial e_{x x}^{0}\right)\left(u^{\prime}-y v^{\prime \prime}-z w^{\prime \prime}-\omega \phi^{\prime \prime}\right) \delta\left(u^{\prime}-y v^{\prime \prime}-z w^{\prime \prime}-\omega \phi^{\prime \prime}\right) \\
& \left.+\frac{1}{2}\left(\partial \sigma_{s x}^{0} / \partial e_{s x}^{0}\right) \boldsymbol{\theta} \phi^{\prime} \delta\left(\boldsymbol{\theta} \phi^{\prime}\right)\right] d v-\boldsymbol{F}^{T} \delta \boldsymbol{d}=0
\end{aligned}
$$

Following the same procedure and notations as in Reference 4), the incremental stiffness equation for the thin-walled straight beam element of inelastic material can be determined in the form

$$
\left(\begin{array}{c}
F_{x} \\
F_{y} \\
F_{z} \\
T
\end{array}\right)=\left[\begin{array}{llll}
K_{11} & & \text { sym } & \\
K_{21} & K_{22} & & \\
K_{31} & K_{32} & K_{33} & \\
K_{41} & K_{42} & K_{43} & K_{44}
\end{array}\right]\left(\begin{array}{c}
U \\
V \\
W \\
\Phi
\end{array}\right)
$$

where

$$
\begin{aligned}
& \boldsymbol{K}_{11}=\int_{\boldsymbol{A}}\left(\partial \sigma_{x x}^{0} / \partial e_{x x}^{0}\right) d a \int_{L}\left(\boldsymbol{A}^{\prime}\right)\left(\boldsymbol{A}^{\prime}\right)^{T} d x \\
& \boldsymbol{K}_{22}=\int_{A}\left(\partial \sigma_{x x}^{0} / \partial e_{x x}^{0}\right) y^{2} d a \int_{L}\left(\boldsymbol{B}^{\prime \prime}\right)\left(\boldsymbol{B}^{\prime \prime}\right)^{T} d x+\int_{A} \sigma_{x x}^{0} d a \int_{L}\left(\boldsymbol{B}^{\prime}\right)\left(\boldsymbol{B}^{\prime}\right)^{T} d x \\
& \boldsymbol{K}_{33}=\int_{A}\left(\partial \sigma_{x x}^{0} / \partial e_{x x}^{0}\right) z^{2} d a \int_{L}\left(\boldsymbol{B}^{\prime \prime}\right)\left(\boldsymbol{B}^{\prime}\right)^{T} d x+\int_{A} \sigma_{x x}^{0} d a \int_{L}\left(\boldsymbol{B}^{\prime}\right)\left(\boldsymbol{B}^{\prime}\right)^{T} d x \\
& \boldsymbol{K}_{44}=\int_{A}\left(\partial \sigma_{x x}^{0} / \partial e_{x x}^{0}\right) \omega^{2} d a \int_{L}\left(\boldsymbol{B}^{\prime \prime}\right)\left(\boldsymbol{B}^{\prime \prime}\right)^{T} d x+\int_{A} \sigma_{x x}^{0}\left(y^{2}+z^{2}\right) d a \int_{L}\left(\boldsymbol{B}^{\prime}\right)\left(\boldsymbol{B}^{\prime}\right)^{T} d x
\end{aligned}
$$




$$
\begin{aligned}
& +\int_{A} \frac{1}{2}\left(\partial \sigma_{s x}^{0} / \partial e_{s x}^{0}\right) \boldsymbol{\theta}^{2} d a \int_{L}\left(\boldsymbol{B}^{\prime}\right)\left(\boldsymbol{B}^{\prime}\right)^{T} d x \\
& \boldsymbol{K}_{21}=-\int_{A}\left(\partial \sigma_{x x}^{0} / \partial e_{x x}^{0}\right) y d a \int_{L}\left(\boldsymbol{B}^{\prime \prime}\right)\left(\boldsymbol{A}^{\prime}\right)^{r} d x \\
& \boldsymbol{K}_{31}=-\int_{A}\left(\partial \sigma_{x x}^{0} / \partial e_{x x}^{0}\right) z d a \int_{L}\left(\boldsymbol{B}^{\prime \prime}\right)\left(\boldsymbol{A}^{\prime}\right)^{T} d x \\
& \boldsymbol{K}_{32}=\int_{A}\left(\partial \sigma_{x x}^{0} / \partial e_{x x}^{0}\right) y z d a \int_{L}\left(\boldsymbol{B}^{\prime \prime}\right)\left(\boldsymbol{B}^{\prime \prime}\right)^{T} d x \\
& \boldsymbol{K}_{\mathbf{4}}=-\int_{A}\left(\partial \sigma_{x x}^{0} / \partial e_{x x}^{0}\right) \omega d a \int_{L}\left(\boldsymbol{B}^{\prime \prime}\right)\left(\boldsymbol{A}^{\prime}\right)^{T} d x \\
& \boldsymbol{K}_{42}=\int_{\boldsymbol{A}}\left(\partial \sigma_{x x}^{0} / \partial e_{x x}^{0}\right) y \omega d a \int_{L}\left(\boldsymbol{B}^{\prime \prime}\right)\left(\boldsymbol{B}^{\prime \prime}\right)^{T} d x+\int_{\boldsymbol{A}} \sigma_{x x}^{0} z d a \int_{L}(\boldsymbol{B})\left(\boldsymbol{B}^{\prime}\right)^{T} d x \\
& \boldsymbol{K}_{43}=\int_{A}\left(\partial \sigma_{x x}^{0} / \partial e_{x x}^{0}\right) z \omega d a \int_{L}\left(\boldsymbol{B}^{\prime \prime}\right)\left(\boldsymbol{B}^{\prime \prime}\right)^{T} d x-\int_{A} \sigma_{x x}^{0} y d a \int_{L}(\boldsymbol{B})\left(\boldsymbol{B}^{\prime \prime}\right)^{T} d x
\end{aligned}
$$

The block stiffness matrices given in Eq. (7) can be re-written in reduced forms as

$$
\begin{aligned}
& K_{11}=(1 / L) \int_{A}\left(\partial \sigma_{x x}^{0} / \partial e_{x x}^{0}\right) d a K_{0} \\
& K_{22}=\left(1 / L^{3}\right) \int_{A}\left(\partial \sigma_{x x}^{0} / \partial e_{x x}^{0}\right) y^{2} d a K_{1}+(1 / L) \int_{A} \sigma_{x x}^{0} d a K_{2} \\
& K_{33}=\left(1 / L^{3}\right) \int_{A}\left(\partial \sigma_{x x}^{0} / \partial e_{x x}^{0}\right) z^{2} d a K_{1}+(1 / L) \int_{A} \sigma_{x x}^{0} d a K_{2} \\
& K_{44}=\left(1 / L^{3}\right) \int_{A}\left(\partial \sigma_{x x}^{0} / \partial e_{x x}^{0}\right) \omega^{2} d a K_{1}+(1 / L) \int_{A} \sigma_{x x}^{0}\left(y^{2}+z^{2}\right) d a K_{2} \\
& +(1 / L) \int_{A} \frac{1}{2}\left(\partial \sigma_{s x}^{0} / \partial e_{s x}^{0}\right) \Theta^{2} d a K_{2} \\
& K_{21}=-\left(1 / L^{2}\right) \int_{A}\left(\partial \sigma_{x x}^{0} / \partial e_{x x}^{0}\right) y d a K_{6} \\
& K_{31}=-\left(1 / L^{2}\right) \int_{A}\left(\partial \sigma_{x x}^{0} / \partial e_{x x}^{0}\right) z d a K_{6} \\
& K_{32}=\left(1 / L^{3}\right) \int_{A}\left(\partial \sigma_{x x}^{0} / \partial e_{x x}^{0}\right) y z d a K_{1} \\
& K_{41}=-\left(1 / L^{2}\right) \int_{A}\left(\partial \sigma_{x x}^{0} / \partial e_{x x}^{0}\right) \omega d a K_{6} \\
& K_{42}=\left(1 / L^{3}\right) \int_{A}\left(\partial \sigma_{x x}^{0} / \partial e_{x x}^{0}\right) y \omega d a K_{1}+(1 / L) \int_{A} \sigma_{x x}^{0} z d a K_{3} \\
& K_{43}=\left(1 / L^{3}\right) \int_{A}\left(\partial \sigma_{x x}^{0} / \partial e_{x x}^{0}\right) z \omega d a K_{1}-(1 / L) \int_{A} \sigma_{x x}^{0} y d a K_{3}
\end{aligned}
$$

in which $K_{0} \sim K_{3}$ are the integration matrices defined in Reference 4 ), and $K_{6}$ is given by

$$
\boldsymbol{K}_{6}=\left[\begin{array}{cc}
0 & 0 \\
-L & L \\
0 & 0 \\
L & -L
\end{array}\right]
$$

The current strain at an arbitrary point on the cross-section can be calculated in terms of the known displacement derivatives using the relation

$$
e_{x x}^{0}=\varepsilon^{0}+y x_{y}^{0}+z x_{z}^{0}+\omega x_{\omega}^{0}
$$

Hence, by making use of the relation between axial stress and strain, it is possible to calculate $\sigma_{x x}^{0}$ and $\partial \sigma_{x x}^{0} / \partial e_{x x}^{0}$ across the section and thus all the block stiffness expressions appeared in the stiffness matrix can be computed. As is clear, those quantities are to be calculated for each and every element, at each incremental step.

As is explained in Eq. $(1 \cdot \mathrm{b})$, when calculating the term $\int_{A}(1 / 2)\left(\partial \sigma_{s x}^{0} / \partial e_{s x}^{0}\right) \theta^{2} d a$, it is assumed that $\partial \sigma_{s x}^{0} / \partial e_{s x}^{0}$ is reduced from its original value at the unstressed state by the same ratio as that of the axial stress. Hence it can be computed by making use of the relation 
$\int_{A}\left(\partial \sigma_{s x}^{0} / \partial e_{s x}^{0}\right) \theta^{2} d a=2 \frac{G}{E} \int_{A}\left(\partial \sigma_{x x}^{0} / \partial e_{x x}^{0}\right) \theta^{2} d a$

\section{EVALUATIONS OF STRAIN AND STRESS INCREMENTS}

After solving the incremental equation, the increments in the element end forces can be found by resubstitution of the displacement increments to the individual element stiffness matrices, and hence the new internal stress resultants can be determined by adding the increments to the previous element end forces and transforming the total end forces to the local coodinates, simply following a similar incremental procedure introduced in Reference 5). However, in calculation of the block stiffness expressions of Eqs. ( 8 ) in the incremental stiffness matrix, a special procedure has to be followed to find the corresponding increments in the displacement derivatives. For this purpose, the linear equations relating the increments of stress resultants to those of the displacement derivatives are derived in the following way.

Increment in the axial strain is related to the incremental displacement derivatives by the equation as

$$
\Delta e_{x x}^{0}=\Delta \varepsilon^{0}+y \Delta x_{y}^{0}+z \Delta x_{z}^{0}+\omega \Delta x_{\omega}^{0}
$$

Therefore

$$
\Delta \sigma_{x x}^{0}=\left(\partial \sigma_{x x}^{0} / \partial e_{x x}^{0}\right) \Delta e_{x x}^{0}=\left(\partial \sigma_{x x}^{0} / \partial e_{x x}^{0}\right)\left(\Delta \varepsilon^{0}+y \Delta x_{y}^{0}+z \Delta x_{z}^{0}+\omega \Delta x_{\omega}^{0}\right)
$$

and hence

$$
\Delta N^{0}=\int_{A} \Delta \sigma_{x x}^{0} d a=\int_{A}\left(\partial \sigma_{x x}^{0} / \partial e_{x x}^{0}\right)\left(\Delta \varepsilon^{0}+y \Delta x_{y}^{0}+z \Delta x_{z}^{0}+\omega \Delta \varkappa_{\omega}^{0}\right) d a
$$

Similarly

$$
\begin{aligned}
& \Delta M_{y}^{0}=\int_{A}\left(\partial \sigma_{x x}^{0} / \partial e_{x x}^{0}\right) y\left(\Delta \varepsilon^{0}+y \Delta x_{y}^{0}+z \Delta x_{z}^{0}\right. \\
& \left.+\omega \Delta x_{\omega}^{0}\right) d a \\
& \Delta M_{z}^{0}=\int_{A}\left(\partial \sigma_{x x}^{0} / \partial e_{x x}^{0}\right) z\left(\Delta \varepsilon^{0}+y \Delta x_{y}^{0}+z \Delta x_{z}^{0}\right. \\
& \left.+\omega \Delta x_{\omega}^{0}\right) d a \\
& \Delta M_{\omega}^{0}=\int_{A}\left(\partial \sigma_{x x}^{0} / \partial e_{x x}^{0}\right) \omega\left(\Delta \varepsilon^{0}+y \Delta x_{y}^{0}+z \Delta x_{z}^{0}\right. \\
& \left.+\omega \Delta x_{\omega}^{0}\right) d a
\end{aligned}
$$

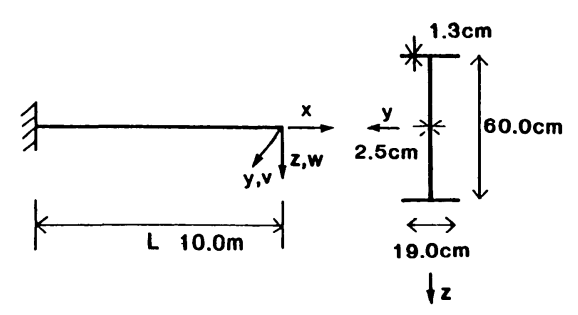

Fig. 2 A Cantilever Beam.

The relations above can be expressed in matrix form as

$$
\Delta \boldsymbol{P}=\mathbf{S} \Delta \boldsymbol{D}
$$

where

$$
\begin{aligned}
& \Delta \boldsymbol{P}=\left\langle\Delta N^{0}, \Delta M_{y}^{0}, \Delta M_{z}^{0}, \Delta M_{\omega}^{0}\right\rangle^{T} \\
& \Delta D=\left\langle\Delta \varepsilon^{0}, \Delta x_{y}^{0}, \Delta x_{z}^{0}, \Delta x_{\omega}^{0}\right\rangle^{T} \ldots \ldots
\end{aligned}
$$
and

$$
\boldsymbol{S}=\int_{A}\left(\partial \sigma_{x x}^{0} / \partial e_{x x}^{0}\right) \boldsymbol{H H}^{T} d a
$$
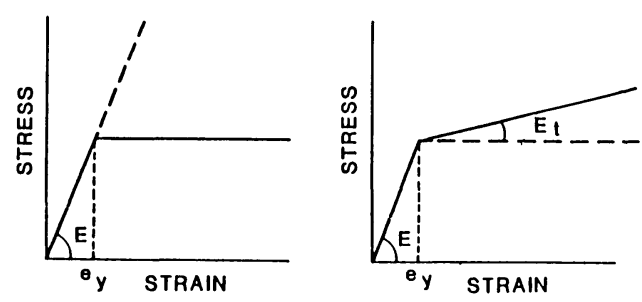

Fig. 3 Stress-Strain Relations.

in which

$$
\boldsymbol{H}=\langle 1, y, z, \omega\rangle^{T} \text {. }
$$

Solving Eq. (15) leads to the increments in the displacement derivatives and thus the increments in the axial stain can be computed. Hence, by using the tangent modulus for axial stress, the increments in axial stress can be found. Also, if required, the increments in the shear stress can be computed by means of taking the equilibrium of an infinitesimal element with the axial stress, as expressed by

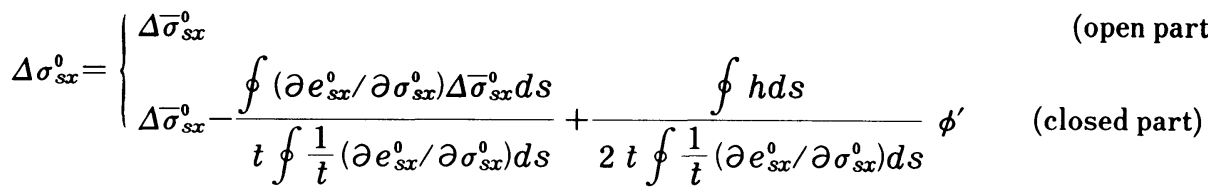


in which

$$
\begin{aligned}
\Delta \bar{\sigma}_{s x}^{0}= & -\left[\frac{1}{t} \int_{0}^{s}\left(\partial \sigma_{x x}^{0} / \partial e_{x x}^{0}\right) \boldsymbol{H} t d s\right]^{T} \\
& \cdot \boldsymbol{S}^{-1} \Delta \boldsymbol{P}^{\prime} \ldots \ldots \ldots \ldots \ldots \ldots \ldots \ldots \ldots \ldots \ldots \ldots \ldots \ldots \ldots
\end{aligned}
$$

where the variation of shear in the $n$-direction is neglected because of its minor contribution, and the coordinate $s=0$ corresponds to an open end for cross-sections with open part.

After all, the shear as well as axial stresses for next iteration step can be evaluated by accumulating those increments obtained at present state. If an appropriate yield criterion will be introduced, the constitutive equation (1) can be readily applicable for continuously changing tangent moduli of the material concerned.

\section{ILLUSTRATIVE EXAMPLES AND DISCUSSIONS}

To demonstrate the procedure stated above, the following constitutive equation is considered as

$$
\frac{\partial \sigma_{x x}^{0}}{\partial e_{x x}^{0}}=\left\{\begin{array}{l}
E\left(\sigma_{e q}<\sigma_{y}\right) \\
E_{t}\left(\sigma_{e q} \geq \sigma_{y}\right)
\end{array}\right.
$$

where $E_{t}$ is the constant tangent modulus after yielding, and $\sigma_{e q}$ is the equivalent stress obtained from the Mises yield criterion, and is expressed as

$$
\sigma_{e q}=\sqrt{\sigma_{x x}^{2}+3 \sigma_{s x}^{2}}
$$

which can be transformed using Eq. $(1 \cdot b)$ to the strain expression of

$$
e_{e q}=\sqrt{e_{x x}^{2}+12(G / E)^{2} e_{s x}^{2}}
$$

Considered for numerical demonstration is a cantilever beam with I-section, as shown in Fig.2, with the uniaxial stress-strain relations, as indicated by Fig. 3 . Since the real behavior or experiments are difficult to trace the very large displacements and also largely depend on their own material properties, the comparison with them are not the objectives of the present computations. But the present

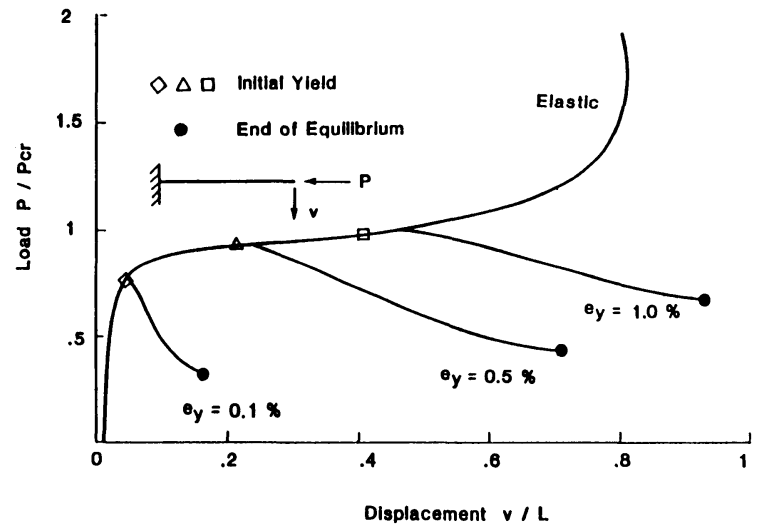

Fig. 4 In-Plane Behaviour of a Cantilever Beam.

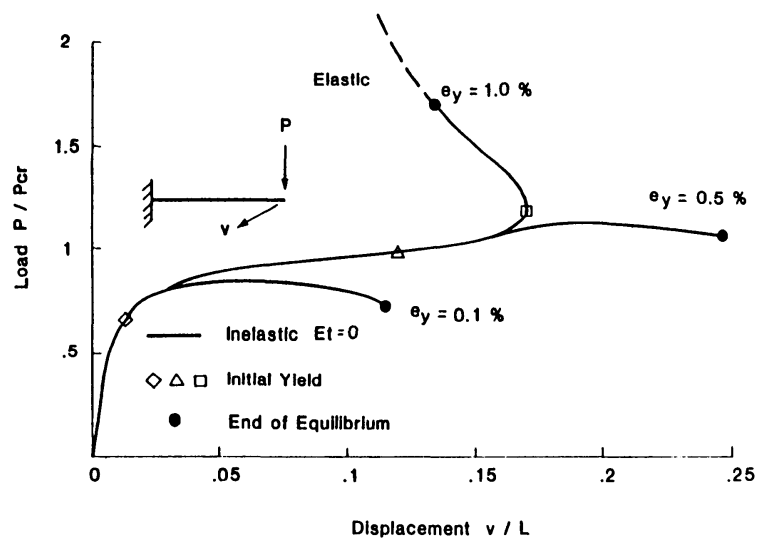

Fig. 5 Spatial Behaviour of a Cantilever Beam with $E_{t}=0$.

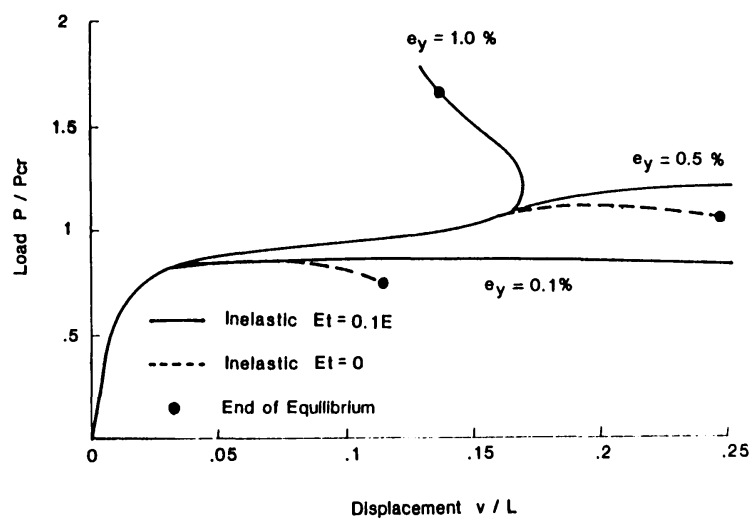

Fig. 6 Spatial Behaviour of a Cantilever Beam with $E_{t}=0.1 E$ examples are just to demonstrate the proposed analysis scheme, showing some general interesting features of the inelastic finite displacement behaviour of thin-walled structures.

The following two cases for how to introduce the Mises yield criterion are examined. All the computations made below assume $E=2.06 \times 10^{5} \mathrm{MPa}$ and $G=7.95 \times 10^{4} \mathrm{MPa}$, and the number of elements are eight.

(1) Only with axial stress contribution: $\sigma_{e q}=\sigma_{x x}$ 
Since the axial stress $\sigma_{x x}$ plays the most important role on the beam mechanics, the shear stress obtained from Eqs. (19) is ignored for the first case, for the simplicity and comparison purposes. Fig. 4 gives the example of the in-plane behaviour with $E_{t}=0$, showing the relation for three different values of yield strains between axial force and lateral diplacement. In the figure, $P_{c r}$ is the in-plane buckling load, and the in-plane disturbing tip force $P_{0}=0.01 P_{c r}$ was given at the initial stage. It is noted that, firstly, the initial yield load does not differ much from the true ultimate load, and secondly, the end of equilibrium, after which the equilibrium path could not be found anyway, exists for this particular case of $E_{t}=0$. With the same condition as the previous example, the out-of-plane spatial behaviour is examined, and obtained in Fig. 5, showing the relation between lateral force and out-pf-plane desplacement. In the figure, $P_{c r}$ is the out-of-plane buckling load, and the disturbing torsional moment $T_{0}=6.69 \times 10^{-3}$ $P_{c r} L$ was given at the initial stage. A similar feature is observed as the in-plane behavior but with noticeable difference between the initial yield and ultimate loads, particularly when the yield strain is large. Fig. 6 shows also the spatial behaviour of the same problem but with the tangent modulus $E_{t}=0.1 E$, in comparison with the previous case of $E_{t}=0$. It is noted that the end of equilibrium is not observed, that is, there would always exist equilibrium for any displaced configuration, just as seen in the elastic problem.

(2) Shear stress combined with axial stresses : $\sigma_{e q}=\sqrt{\sigma_{x x}^{2}+3 \sigma_{s x}^{2}}$

Since the deformation due to the shear stress in equilibrium with the axial stress is neglected in the beam mechanics, the shear stress itself seems rather supplementary, and then does not cause much influences on the beam-behaviour as far as elasticity is concern-

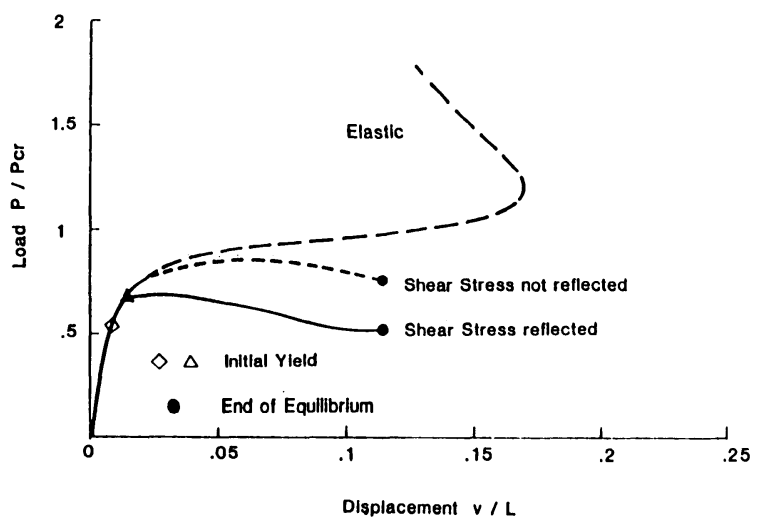

Fig. 7(a) Effects of Shear stresses on Spatial Behaviour of a Cantilever Beam with $E_{t}=0$ and $e_{y}=0.1 \%$.

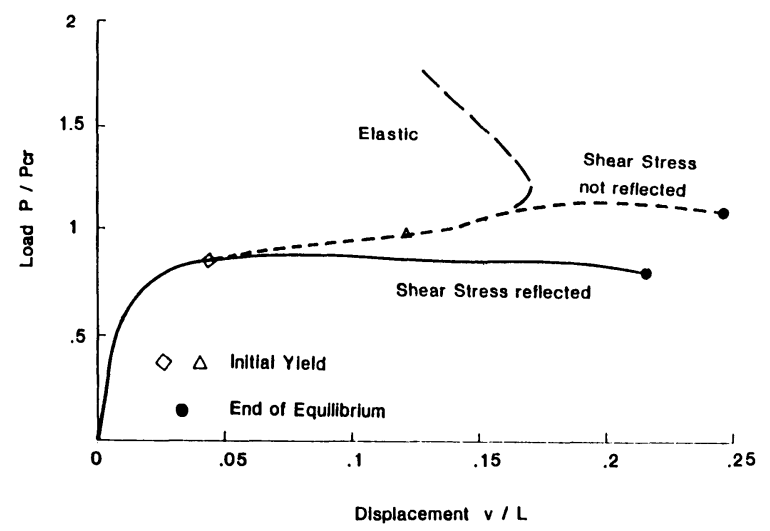

Fig. 7(b) Effects of Shear Stresses on Spatial Behaviour of a Cantilever Beam with $E_{t}=0$ and $e_{y}=0.5 \%$.

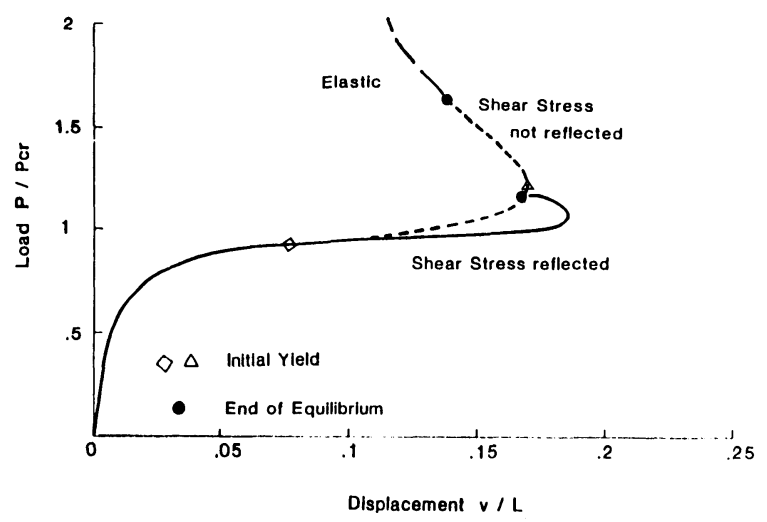

Fig. 7(c) Effects of Shear Stresses on Spatial Behaviour of a Cantilever Beam with $E_{t}=0$ and $e_{y}=1.0 \%$. ed. When inelasticity is involved, however, the shear stress is a primary factor for the Mises yield criterion, leading to a remarkable effect on the inelastic behaviour. Fig. 7 shows the results of computation for the same example as Fig. 5, indicating the effects of shear stresses on the spatial behaviour of the cantilever with $E_{t}=0$. For all the three different values of yield strains, it is evident that the inclusion of 
shear stresses on the Mises yield criterion gives naturally a remarkable difference on the initial yield load, leading to a significant difference on the inelastic load-displacement behaviour.

\section{SUMMARY AND CONCLUSIONS}

Based on the second order kinemateic field, the general stiffness equation of linearized finite desplacements for a thin-walled member with inelastic material has been formulated, using the theorem of virtual work. The stiffness equation is in a simillar form as in the case of elastic material, with additional block stiffness expressions appearing due to the nonlinearity of the material. Also, unlike elastic material, the block stiffness expressions are in terms of area integrals involving axial stress, and tangent moduli for axial and shear stresses, which correspond to the stress resultants, Young's and shear moduli respectively for elastic material.

The direct solution of the stiffness equation is adopted to obtain the nonlinear finite desplacement behaviour of thin-walled beams with inelastic material, using an updating procedure available in Reference 5). An incremental approach is adopted in updating the strains, stresses and the tangent moduli across the cross section also.

Numerilcal examples are presented for the spatial as well as in-plane nonlinear load-displacement behaviour of thin-walled beams with inelastic material. It has been noticed through the numerical examples that the effect of shear stress resulting from the equilibrium with axial stresses towards the member axis is significant, and thus it should be incorporated in the yield condition, even though the shear deformation itself is naturally neglected in the incremental formulations of beam mechanics. Compared with the existing analysis procedures for structures with inelastic material, the present scheme has assumed a rather simplified constitution equation, and partly by virtue of this, seems efficient and versatile, helped by its concise and explicit expressions. It remains to be further investigated, however, that some appropriate modifications need to be introduced within the frame work of the present formulation in order to predict well the real inelastic finite displacement behaviour of thin-walled members.

\section{ACKNOWLEDGEMENT}

This study is suppoted in part by the Grant-in-Aid for Scientific Research from the Japanese Ministry of Education;, Scinece and Culture.

\section{REFERENCES}

1) Rajasekaran, S. and Murray, D. W. : Finite Element Solution of Inelastic Beam Equations, Journal of the Structural Division, ASCE, Vol.99, No. ST6, pp. 1025 1041, June 1973.

2) Murray, D. W. and Rajasekaran, S. : Technique for Formulating Beam Equations, Journal of Engineering Mechanics Division, ASCE, Vol. 101, No. EM5, pp. 561 573, October 1975.

3) Sakimoto, T., Yamao, T., Kikuchi, R. and Sakata, T. : Nonlinear Analysis of Thin-Walled Frames and Members with Arbitrary Open Cross Sections, Structural Engineering/Earthquake Engineering, Vol. 2, No. 2, pp. 385s 393s, Japan Society of Civil Engineers (Proc. of JSCE, No.362/I -4), October 1985.

4) Hasegawa, A., Liyanage, K., Ikeda, T. and Nishino, F. : A Concise and Explicit Formulation of Out-of-Plane Instability of Thin-Walled Members, Structural Engineering/Earthquake Engineering, Vol.2, No. 1, pp. 57s-65s, Japan Society of Civil Engineers (Proc. of JSCE, No. 356/ I -3), April 1985.

5) Hasegawa, A., Liyanage, K. K. and Nishino, F. : A Non-Iterative Nonlinear Analysis Scheme of Space Frames with Thin-Walled Elastic Members, Structural Engineering/Earthquake Engineering, Vol.4, No. 1 pp. 19s 29s, Japan Society of Civil Engineers(Proc. of JSCE, No. 380/ I -7), April 1987. 